\title{
Analysis of the hybrid genomes of two field isolates of the soil-borne fungal species Verticillium longisporum
}

\author{
Johan Fogelqvist', Georgios Tzelepis ${ }^{1}$, Sarosh Bejai ${ }^{1}$, Jonas Ilbäck ${ }^{1,2}$, Arne Schwelm $^{1}$ and Christina Dixelius ${ }^{1 *}$ (D)
}

\begin{abstract}
Background: Brassica plant species are attacked by a number of pathogens; among them, the ones with a soilborne lifestyle have become increasingly important. Verticillium stem stripe caused by Verticillium longisporum is one example. This fungal species is thought to be of a hybrid origin, having a genome composed of combinations of lineages denominated $\mathrm{A}$ and $\mathrm{D}$. In this study we report the draft genomes of $2 \mathrm{~V}$. longisporum field isolates sequenced using the Illumina technology. Genomic characterization and lineage composition, followed by selected gene analysis to facilitate the comprehension of its genomic features and potential effector categories were performed.
\end{abstract}

Results: The draft genomes of 2 Verticillium longisporum single spore isolates (VL1 and VL2) have an estimated ungapped size of about $70 \mathrm{Mb}$. The total number of protein encoding genes identified in VL1 was 20,793, whereas 21,072 gene models were predicted in VL2. The predicted genome size, gene contents, including the gene families coding for carbohydrate active enzymes were almost double the numbers found in $V$. dahliae and $V$. albo-atrum. Single nucleotide polymorphisms (SNPs) were frequently distributed in the two genomes but the distribution of heterozygosity and depth was not independent. Further analysis of potential parental lineages suggests that the $V$. longisporum genome is composed of two parts, A1 and D1, where A1 is more ancient than the parental lineage genome D1, the latter being more closer related to $V$. dahliae. Presence of the mating-type genes MAT1-1-1 and MAT1-2-1 in the $V$. longisporum genomes were confirmed. However, the MAT genes in $V$. dahliae, $V$. albo-atrum and $V$. longisporum have experienced extensive nucleotide changes at least partly explaining the present asexual nature of these fungal species.

Conclusions: The established draft genome of $V$. longisporum is comparatively large compared to other studied ascomycete fungi. Consequently, high numbers of genes were predicted in the two $V$. longisporum genomes, among them many secreted proteins and carbohydrate active enzyme (CAZy) encoding genes. The genome is composed of two parts, where one lineage is more ancient than the part being more closely related to $V$. dahliae. Dissimilar mating-type sequences were identified indicating possible ancient hybridization events.

Keywords: Brassica napus, Carbohydrate active enzymes, Mating-type genes, Verticillium longisporum

\section{Background}

The Verticillium genus belongs to the Plectosphaerellaceae family in the Sordariomycetes, one of the largest classes in Ascomycota. The genus name derives from the "verticilliate" morphological feature of the branched conidiophore, the hyphal structure that bears conidia.

\footnotetext{
* Correspondence: Christina.Dixelius@slu.se

'Department of Plant Biology, Uppsala BioCenter, Linnean Center for Plant Biology, Swedish University of Agricultural Sciences, P.O. Box 7080, 75007 Uppsala, Sweden

Full list of author information is available at the end of the article
}

Verticillium species incite vascular wilt diseases in many crops and wild plant species $[1,2]$. No sexual stage has been reported so far that could help understanding speciation processes and adaptation to different ecological niches and range of plant hosts. Verticillium longisporum substantially shares the disease cycle characteristics with the more studied $V$. dahliae. As $V$. longisporum causes stunting but not wilting in infected plants it has been suggested to change Verticillium wilt to Verticillium stem stripe for the disease [3]. Further, the species name "longisporum" refers to the close to twice as long 
conidia found in most strains in comparison to $V$. dahliae [4-6]. This fungal species is known to have narrower host range compared to $V$. dahliae with preference for host species within the family Brassicaceae, including Brassica species and Arabidopsis thaliana [7, 8]. A recent study suggests a somewhat wider host-range [9]. In Sweden, sugar beet is not known as a host to $V$. longisporum but to V. dahliae [10].

Winter oilseed rape (Brassica napus) is the most important oil crop in Europe and the demand of products from this crop is increasing, not least in various new innovative and bioeconomical contexts. $V$. longisporum has become an important pathogen in most European countries, particularly in regions with intense oilseed rape production [3]. Spread of this plant pathogen is also reported from Canada (http://www.inspection.gc.ca/ plants/plant-pests-invasive-species/diseases/verticilliumwilt/). A major problem is the longevity of its microsclerotia that are released into the soil from infected plant residues at the end of the disease cycle. These resting structures have the capacity to remain dormant in soil for many years. The disease cycle of $V$. longisporum is still unclear at certain parts but it is thought that the microsclerotia are stimulated to germinate via root exudates released from a host plant growing nearby. The hyphae then invade the lateral roots and root hairs followed by colonization of the root tissues and finally enter into the xylem elements. Next, conidia form and these spores can spread via the plant's transpiration stream. Thus conidia are able to colonize vessel tissue further up in the plant, processes which could interfere with access to the xylem sap potentially affecting plant growth. Because the microsclerotia do not form and protrude stems and leaves until the plant is in the senescence phase, the disease infection easily remains unnoticed.

More than 50 years ago it was reported that some Verticillium strains had twice as long conidia and roughly double amount of nuclear DNA as those of $V$. dahliae [11]. Later, similar observations on the diploid status of a potentially new Verticillium species were published $[4,12]$. Population genetic and phylogenetic studies supported that $V$. longisporum is a distinct species. This conclusion was based on restriction length fragment polymorphism (RLFP) and amplified fragment length polymorphism (AFLP) analyses, together with sequence data of mitochondrial and nuclear genes on a high number of fungal isolates $[6,13,14]$. The phylogenetic data indicated a closer relationship between $V$. longisporum, $V$. dahliae and $V$. albo-atrum, compared to $V$. tricorpus and the distantly related $V$. nigrescens, now placed in the Gibellulopis genus [15]. Further studies based on intron-rich sequences of five protein-encoding genes and ribosomal internal transcribed spacer sequences suggested that $V$. longisporum is a hybrid between $V$. dahliae and unknown lineages [16-18]. The progenitors were named A1 and D1, D2 and D3 all together resulting in $3 \mathrm{~V}$. longisporum lineage compositions A1/D1, A1/D2 and A1/D3. The A1 and D1 progenitors were reported to be of unknown origin whereas D2 and D3 were suggested to represent $2 \mathrm{~V}$. dahliae lineages. For further background and details on this fungus, see reviews by Depotter et al. [3, 19].

In the present study we aimed to establish new information on the $V$. longisporum genome composition by sequencing two fungal strains (VL1 and VL2), and to compare the data with genomes from three other Verticillium species; $V$. dahliae, V. albo-atrum and V. tricorpus [20, 21].

\section{Results}

\section{Mapping and SNP analysis}

First, Illumina sequence data generated from DNA of the $2 \mathrm{~V}$. longisporum strains were mapped to the PacBio sequences of $V$. dahliae [22]. Most of the $V$. longisporum paired-end reads could be consistently mapped to the $V$. dahliae reference genome (86 and 79\% in VL1 and VL2, respectively) and most of the $V$. dahliae reference genome was covered by $V$. longisporum reads, 93\% (VL1) and $94 \%$ (VL2). The distribution of coverage was bimodal in both $V$. longisporum strains, representing reads originating from one or both parental genomes, with peaks at $100 \times$ and $200 \times$ for VL1 and $75 \times$ and $150 \times$ for VL2 (Additional file 1). Single nucleotide polymorphisms (SNPs) were distributed, on average one in $24.37 \mathrm{bp}$ (VL1) and one in $23.81 \mathrm{bp}$ (VL2), in total 1,404,670 and $1,438,074$ SNPs, respectively. The vast majority of SNPs was heterozygous, $84.7 \%$ in VL1 and $84.6 \%$ in VL2. The distribution of heterozygosity and depth was not independent, as expected considering a hybrid genomic constitution of this species. The heterozygosity of SNPs with a depth of coverage corresponding the largest peak in the bimodal coverage distribution was about 0.92 in both VL1 and VL2, whereas SNPs with a depth corresponding to the smaller peak had a heterozygosity of about 0.5 (Additional file 1).

\section{Evolution of the $V$. longisporum genome}

We looked at the parental lineage origin at the coding sequence level in our data sets because of the suggested composition of A and D sub-genomes in the genome of V. longisporum [16]. We successfully reconstructed 639 and 668 regions in VL1 and VL2, with an average length of $29.0 \pm 17.4$ and $29.3 \pm 18.7 \mathrm{~kb}$. In total 6471 (VL1) and 6788 (VL2) transcripts were successfully phased, which is 56 and $59 \%$ of the total number of transcripts in $V$. dahliae. The distribution of the fourfold degenerate transversion (4DTv)-rate was different when comparing $V$. dahliae to each of the parental genomes of VL1 and 
VL2. For both VL1 and VL2 there was a clear peak at $4 \mathrm{DTv}=0.006$ in the comparison between $V$. dahliae and the $\mathrm{D}$ parental genome, whereas there was a peak at $4 \mathrm{DTv}=0.05$ for the comparison between VD and the A parental genome as well as for comparing the A to the D parental genome (Additional file 2). When comparing the A and D between VL1 and VL2 there were peaks at $4 \mathrm{DTv}=0.003$ for both parental genomes. This suggests a close relationship between $V$. dahliae and the D parental genome. Further, from the collinear block analysis among the genomes of $V$. dahliae and $V$. albo-atrum (and the outgroup $V$. tricorpus), in total 7244 genes could unambiguously be assigned as orthologous, present in exactly one copy in each genome. Successfully reconstructed parental origin in both VL1 and VL2 were possible for 3592 genes. The analysis of 4DTv suggested that $V$. albo-atrum was slightly closer to A than to $\mathrm{D}$, with peaks at about $4 \mathrm{DTv}=0.08$ and 0.09 , respectively (Additional file 2). When we constructed a phylogenetic tree using a concatenation of these genes (3592), $V$. longisporum D was placed close to $V$. dahliae (Additional file 3). Together with $V$. albo-atrum these three genomes formed a monophyletic clade with $V$. longisporum A as a sister group. Whereas the bootstrap values were overall high, gene support frequency and internode certainty values were low for this clade.

\section{Genome characteristics}

The de novo draft genome sequences achieved of the VL1 and VL2 strains consisted of 4620 (VL1) and 6431 (VL2) scaffolds over 500 bp with $\mathrm{N}_{50}$ values of 154,661 (VL1) and 91,201 (VL2) (Table 1). Different assemblers were tried, for example Redundans [23] but no significant improvement in reducing scaffold numbers was achieved compared to the chosen approach. Further, an un-gapped assembly size would reduce the genome size from $95 \mathrm{Mb}$ to $70 \mathrm{Mb}$. Evaluation based of the CEGMA analysis revealed that $94 \%$ (VL1) and 76\% (VL2) of the eukaryotic core genes were full length (98 and $96 \%$ partial) indicating a fragmented assembly. The GC content of the two genomes was 55.8\% (VL1) and 53.3\% (VL2), and the mitochondrial genome sizes were estimated at $27.7 \mathrm{~kb}$ and $26.2 \mathrm{~kb}$, respectively. The total number of protein encoding genes identified in VL1 was 20,793, and 21,072 gene models were predicted in VL2. The majority (13,334 and 12,728) of the predicted gene models could be partitioned into syntenic blocks with $V$. dahliae and/or V. albo-atrum.

Based on intron-rich sequences of: actin, elongation factor 1-alpha, glyceraldehyde-3-phosphate dehydrogenase, mitochondrial oxaloacetate transport protein and tryptophan synthase gene sequences, $V$. longisporum was suggested to be a diploid fungal species generated by
Table 1 Description of genome assemblies of the $2 \mathrm{~V}$. Iongisporum isolates VL1 and VL2

\begin{tabular}{llll}
\hline & & VL1 & VL2 \\
\hline Nuclear genome & Scaffold length (Mb) & $\sim 95$ & $\sim 95$ \\
& Genomic GC & 55.84 & 53.34 \\
& content (\%) & & \\
& Scaffold number & 4620 & 6431 \\
& Scaffold N50 & 154,651 & 91,201 \\
& Scaffold L50 & 150 & 221 \\
& Contig N50 & 26,946 & 73,321 \\
& Contig L50 & 710 & 270 \\
& Number of contigs & 14,286 & 13,586 \\
& Contig length (bp) & $67,181,795$ & $67,661,737$ \\
& Total number of genes & 20,793 & 21,072 \\
Scaffold length (bp) & 27,669 & 26,151 \\
GC content (\%) & 27.13 & 27.82 \\
& Scaffold number & 1 & 1 \\
Number of contigs & 3 & 2 \\
& Contig length (bp) & 26,438 & 26,116 \\
\hline
\end{tabular}

hybridization between three different $\mathrm{D}$ lineages with difference in phylogenetic distance to $V$. dahliae, and the hypothetical ancestor species A1 [16]. By using these five gene sequences including ribosomal internal transcribed spacer (ITS) sequences together with the most similar sequence from VL1 and VL2, we conclude based on phylogenetic reconstruction (Additional file 4A-F) that both our fungal strains were of the A1/D1 type using the nomenclature by Inderbitzin and co-workers [3].

Despite the relatively high numbers of scaffolds achieved, the repeat content in the $2 \mathrm{~V}$. longisporum genomes was low, ranging between 3.13 to $7.87 \%$ of unclassified repeats, and 1.35 to $3.04 \%$ of identified transposable elements (TEs) (Additional file 5). This low level of TEs in $V$. longisporum is consistent with the percentages found in $V$. dahliae and $V$. albo-atrum [20, 24]. To further decipher the potential role of retrotransposons in genomic rearrangements, we performed a combination of RepeatMasker searches and manual inspections of our datasets. Among the retrotransposons identified, the most abundant group was characterized as long terminal repeats (LTR) belonging to class I (Additional file 5). LINE1 was completely absent in VL1, but present in VL2 based on our data. In contrast, VL2 harbored a higher number of LTR/Gypsy elements, 4402 compared to 1338 in VL1. DNA transposons categorized as class II were also more frequent in the VL2 genome, and $h A T$ Ac, TcMar-Pogo and PIF-Harbinger were all present in VL2 but absent in VL1. Copia, Gypsy and Tc1/Mariner are the most widespread transposons in $V$. dahliae and $V$. albo-atrum, known to accumulate in gene-rich 
regions or in dispersed chromosomal spots [25]. No biased locations of TEs in the $2 \mathrm{~V}$. longisporum genomes were however detected in our dataset.

\section{Mating-type genes}

It is known that mating type in Ascomycota is highly variable and consist of dissimilar sequences or idiomorphs [26]. The MAT1-1-1 gene harbors the $\alpha 1$ domain while the MAT1-2-1 gene encodes a transcription factor with a MATA_HMG domain. Due to the hybrid nature of the $V$. longisporum genome we searched for mating type information among the Verticillium species. MAT1-2-1 is annotated in the $V$. dahliae reference genome as VDAG_JR2_Chr2g11860. In contrast, the other idiomorph characterized by the $\alpha 1$-box containing MAT1-1-1 gene is lacking but present in Genbank. MAT-1-1-1 could be identified in $V$. albo-atrum by BLAST search (VDBG_01985T0, E-value 0.0) but no MAT1-2-1 sequence is available.

Searching for homologous genes in our $V$. longisporum genome data revealed two copies of MAT1-1-1 and one copy of MAT1-2-1 in both VL1 and VL2 strains. Typically the two alternate sequences should occupy the same locus on corresponding chromosomes [27]. Here, the MAT1-2-1 sequence was located next to one of the MAT1-1-1 genes in both $V$. longisporum strains, whereas the other copy of MAT1-1-1 was found on a separate contig (Fig. 1). The Verticillium mating type sequences were integrated in a slightly modified multispecies dataset earlier used for the reconstruction of the phylogenetic relationship of the HMG and $\alpha 1$ domain sequences [28]. All MAT1-1-1 and MAT1-2-1 Verticillium domain sequences grouped together with other sequences from Pezizomycotina in Ascomycota (Fig. 2). The single VL MAT1-1-1 copy is more closely related to the $V$. dahliae gene copy (green sub-clade) and probably derived from the $\mathrm{D}$ genome, whereas the VL copy of MAT1-2-1 (yellow sub-clade) is more distant to the $V$. dahliae sequence and by analogy should have derived from the A genome. The presence of MAT1-1-1 and MAT1-2-1 genes in the genome of both $V$. longisporum isolates was confirmed by PCR amplification followed by Sanger sequencing. When more closely analyzing and comparing the complete $M A T$ sequences, and flanking genes, a more complex picture appeared. Overall synteny can be seen but rearrangements have occurred in these chromosomal regions including deletions and inversion/insertion events (Fig. 1). Sequence similarity data further suggests that the MAT genes have been exposed to rather extensive changes. When aligning the sequences using the Pfam database, a loss of the HMG box regulating mating in VDAG_7R2_Chr2g11850 was revealed most likely leading to loss of function in this gene (Additional file 6).

\section{Secretome analysis}

Using a combination of bioinformatics tools, we identified a total of 1281 and 1251 secreted proteins in VL1 and VL2, respectively. This number is higher compared to $V$. dahliae (746) and $V$. albo-atrum (767) but slightly less if the hybrid nature of $V$. longisporum is considered (Additional file 7). Eukaryotic orthologous groups (KOG) analysis of the secreted proteins revealed approximately twice as many proteins involved in ion transport and metabolism, lipid transport and metabolism in VL1 compared to $V$. dahliae and $V$. albo-atrum (Additional file 8). Pathogens secrete a variety of proteins that include potential pathogenicity factors, generally named as effectors [29]. These effectors commonly cysteine-rich proteins are known to suppress or interfere with immune responses of the host metabolism to facilitate pathogen colonization. All the predicted secreted proteins in the $2 \mathrm{~V}$. longisporum strains possessed highly variable numbers of cysteine (Additional files 9, 10 and 11). Both $V$. longisporum genomes were found to harbor fourteen secreted proteins containing a cysteine-rich fungal-specific extracellular EGF-like (CFEM) domain. Four EGF-like gene copies are present in each of the genomes of $V$. dahliae and $V$. albo-atrum [20] but the function of these genes are not understood. By exploiting the proteins predicted as secreted in $V$. longisporum, we were able to detect 204 (VL1) and 203 (VL2) potential effector candidates (Additional file 7). Among these cysteine-rich proteins more than 50\% varied between 200 and 300 amino acids in size. The number of pairwise distances < than $10 \mathrm{~kb}$ was higher than expected among the candidate effectors in both VL1 and VL2 genomes. The effector candidate sequences were clustered to some degree (Additional file 12). The number of pairwise distances among secreted proteins was enriched in the interval $0-20 \mathrm{~kb}$ for VL1 and in the interval of $10-20 \mathrm{~kb}$ in VL2.

Conserved secreted proteins play a significant role for fungal pathogens, and genes coding for LysM effectors residing in the CBM50 peptidoglycan-binding module, and necrosis- and ethylene-inducing-like proteins (NLP) were previously shown to contribute to $V$. dahliae pathogenicity $[24,30]$. Our $V$. longisporum genomes were predicted to host eleven (VL1) and eight (VL2) genes harboring the LysM motif (Additional file 7). NLP-encoding genes are reported to be present in most fungal genomes, and possess cytotoxic activity towards many plant species [31]. In our analysis, we found twelve and fifteen gene homologs in VL1 and VL2 respectively with the necrosis-inducing Phytophthora protein or NPP1-domain, compared to eight in $V$. dahliae and seven in $V$. albo-atrum. 


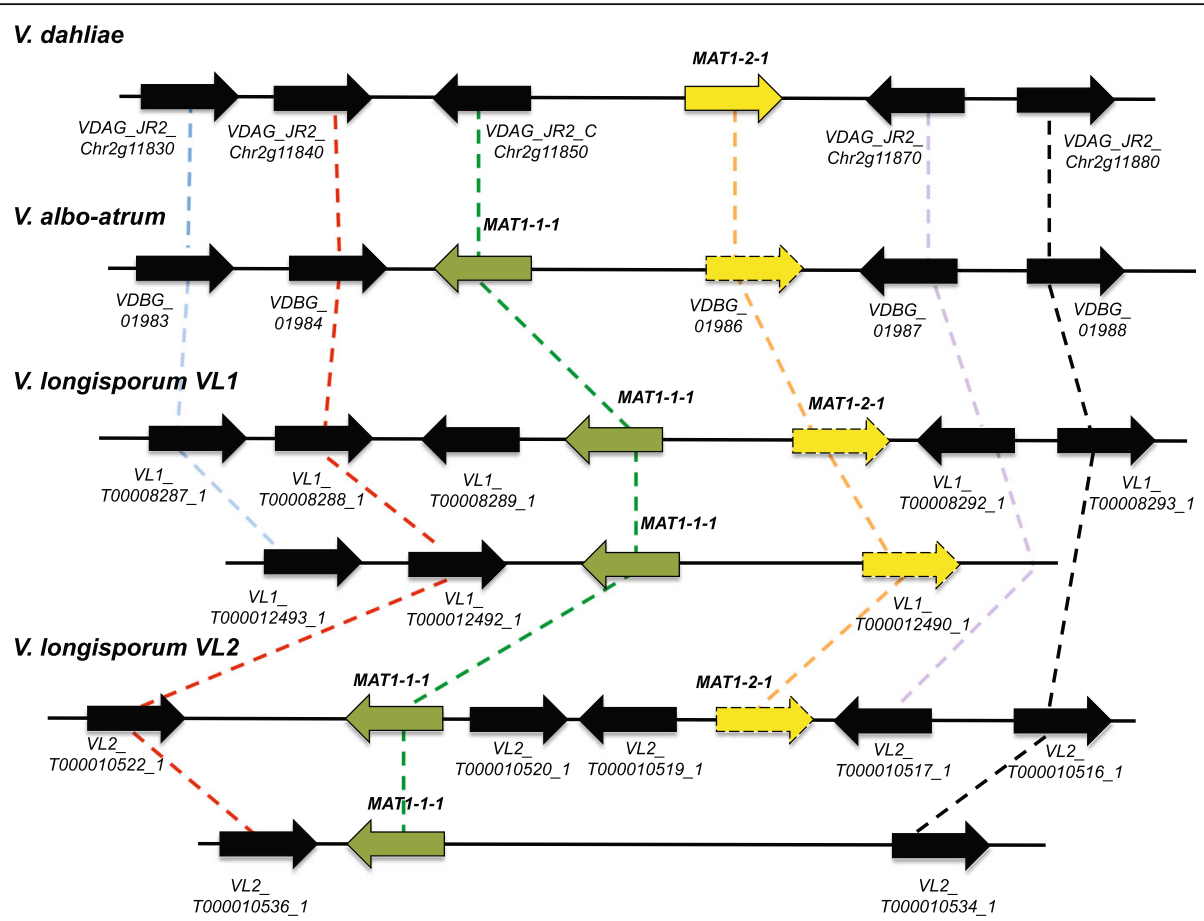

Fig. 1 Genomic organization of mating type genes, in three Verticillium species. In the $V$. longisporum genome sequences the mating type genes were identified on two different contigs in both VL strains. MAT1-1-1 (green), MAT1-2-1 (yellow) and neighboring genes (black). Dashed lines in different colors (blue, red, green, yellow, orange, purple and black) indicate homology among the genes. VDAG_JR2_Chr2g11850 (black) lacks the HMG-box conserved in other MAT1-1-1 proteins. Details on PFAM predictions and levels of sequence homologies are listed in Additional file 6. Gene ID is indicted. The illustration is not drawn to scale

\section{Carbohydrate active-enzymes}

Several studies have demonstrated a strong relationship between the carbohydrate active enzyme (CAZy) repertoire in fungal genomes and their saprophytic, parasitic or necrotrophic life-style strategies [32,33]. The analyses of the two $V$. longisporum genomes revealed a rich repertoire of CAZy families, with close to twice as many when compared to $V$. dahliae, $V$. albo-atrum, and four fungal pathogens known to incite disease on Brassica crops: Alternaria brassicicola, Botrytis cinerea, Leptosphaeria maculans and Sclerotinia sclerotiorum (Fig. 3; Additional file 13). Among the potential effectors $(<400$ amino acids) in the $V$. longisporum genomes, thirteen (VL1) and ten (VL2) contained fungal cellulose-binding domains. The second major sub-group in VL1 and VL2 belonged to the glycosyl hydrolase family with twelve (VL1) and eleven (VL2) proteins. The $V$. dahliae and $V$. albo-atrum genomes are enriched for polysaccharide lyases compared to other sequenced ascomycete fungi [20]. Here we found only ten and nine pectate lyases, respectively, in the two VL genomes with potential effector characteristics (Additional files 9 and 10).

\section{Discussion}

Sordariomycetes (former Pyrenomycetes) is one of the largest classes in the Ascomycota comprising diverse species to which $V$. longisporum belongs [3]. Among others, members of this fungal class are known to cause diseases and produce many important secondary metabolites, some with toxic consequences for animals and humans [34-37]. The family Plectosphaerellaceae harbors a handful of other plant pathogens than Verticillium species, for example the legume pathogen Volutella colletotrichoides that is placed in a related genus [38]. The Verticillium genus has historically harbored a wide range of plant parasites and saprotrophs but has undergone several taxonomic revisions excluding all species except a handful of fungal plant pathogens.

The list of fungal genomes sequenced and publicity available is constantly growing. The fungal genome sizes are presently estimated to range between $8.97 \mathrm{Mb}$ to $117.57 \mathrm{Mb}$, all with high gene diversity and content of repetitive DNA and TEs [39]. The $V$. dahliae and $V$. alboatrum genomes have predicted sizes of $35 \mathrm{Mb}$ and $30 \mathrm{Mb}$, respectively, [20] and the present draft genome of $V$. longisporum has about double this size, which is in line with its proposed hybrid nature. A major force in evolution is genome duplication sometimes involving hybridization, which together are important features of the evolutionary history in many eukaryotic organism groups. Increasing numbers of hybrid species in fungi are described particularly in species such as Saccharomyces, 


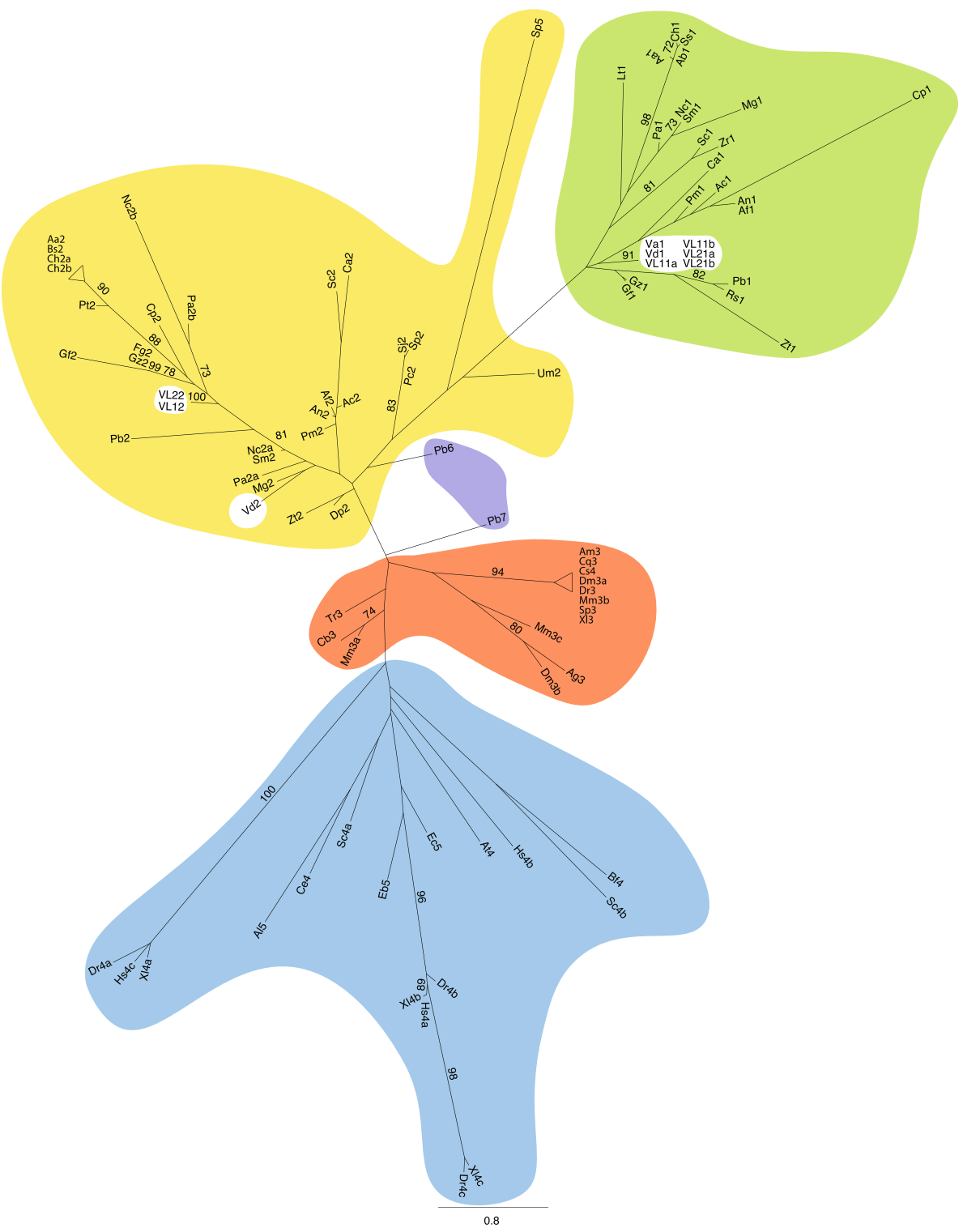

Fig. 2 Maximum likelihood phylogeny (RAXML, model $L G+\Gamma$ ) of the HGM superfamily and the a1 domain core amino acid sequence integrated in a slightly modified dataset [22] now incorporating mating type genes found in V. longisporum strain VL1 and VL2, and V. albo-atrum. Bootstrap support values above 70 are shown. Labeling is as follows: a1 (green), MATA_HMG (yellow), SOX (orange), HMGB (blue), Phycomyces blakesleeanus (Zygomycota), sexM and sexP are circled in purple and Verticillium species (white). The number after the abbreviation indicates the domain as: 1, a1; 2, MATA_HMG; 3, SOX; 4, HMGB; 5, HMG; 6, SeqP and 7, SeqM. In the case more than one domain is present in a species, the suffix a, b, $\mathrm{C}$ is added. Accession numbers for proteins used for the phylogeny is listed in Additional file 14. Species abbreviations: Aa, Alternaria alternata; Ab, Alternaria brassicicola; Ac, l; Af, Aspergillus fumigatus; Ag, Anopheles gambiae; Al, Antonospora locustae; Am, Ailuropoda melanoleuca; An, Aspergillus nidulans; At, Arabidopsis thaliana; Bf, Botryotinia fuckeliana; Bs, Bipolaris sacchari; Ca, Candida albicans; Cb, Cervus elaphus yarkandensis; Ce, Caenorhabditis elegans; Ch, Cochliobolus heterostrophus; Cp, Cryphonectria parasitica; Cq, Culex quinquefasciatus; Cs, Ciona savignyi; Dm, Drosophila melanogaster; Dp, Dothistroma pini; Dr., Danio rerio; Eb, Enterocytozoon bieneusi; Ec, Encephalitozoon cuniculi; Fg, Fusarium acaciae-mearnsii; Gf, Gibberella fujikuroi; Gz, Gibberella zeae; Hs, Homo sapiens; Lt, Lachancea thermotolerans; Mg, Magnaporte grisea; Mm, Mus musculus; Nc, Neurospora crassa; Pa, Podospora anserina; Pb, Pyrenopeziza brassicae; Pc, Pneumocystis carinii; Pm, Penicillium marneffei; Pt, Pyrenophora teres; Rs, Rhynchosporium secalis; Sc, Saccharomyces cerevisiae; Sj, Schizosaccharomyces japonicus; Sm, Sordaria macrospora; Sp, Schizosaccharomyces pombe; Ss, Stemphylium sarciniforme; Tr, Takifugu rubripes; Um, Ustilago maydis; Va, Verticillium albo-atrum; Vd, Verticillium dahliae; VL1, Verticillium longisporum; VL2, Verticillium longisporum; Xl, Xenopus laevis; Zr, Zygosaccharomyces rouxii; Zt, Zymoseptoria tritici

Pichia, Cryptococcus, and Candida [40-44]. Likewise, among plant pathogens, hybridization is increasingly being recognized as an important evolutionary factor [19].
Based on earlier AFLP studies on the European $V$. longisporum population we can conclude that Swedish and German $V$. longisporum isolates from oilseed rape 

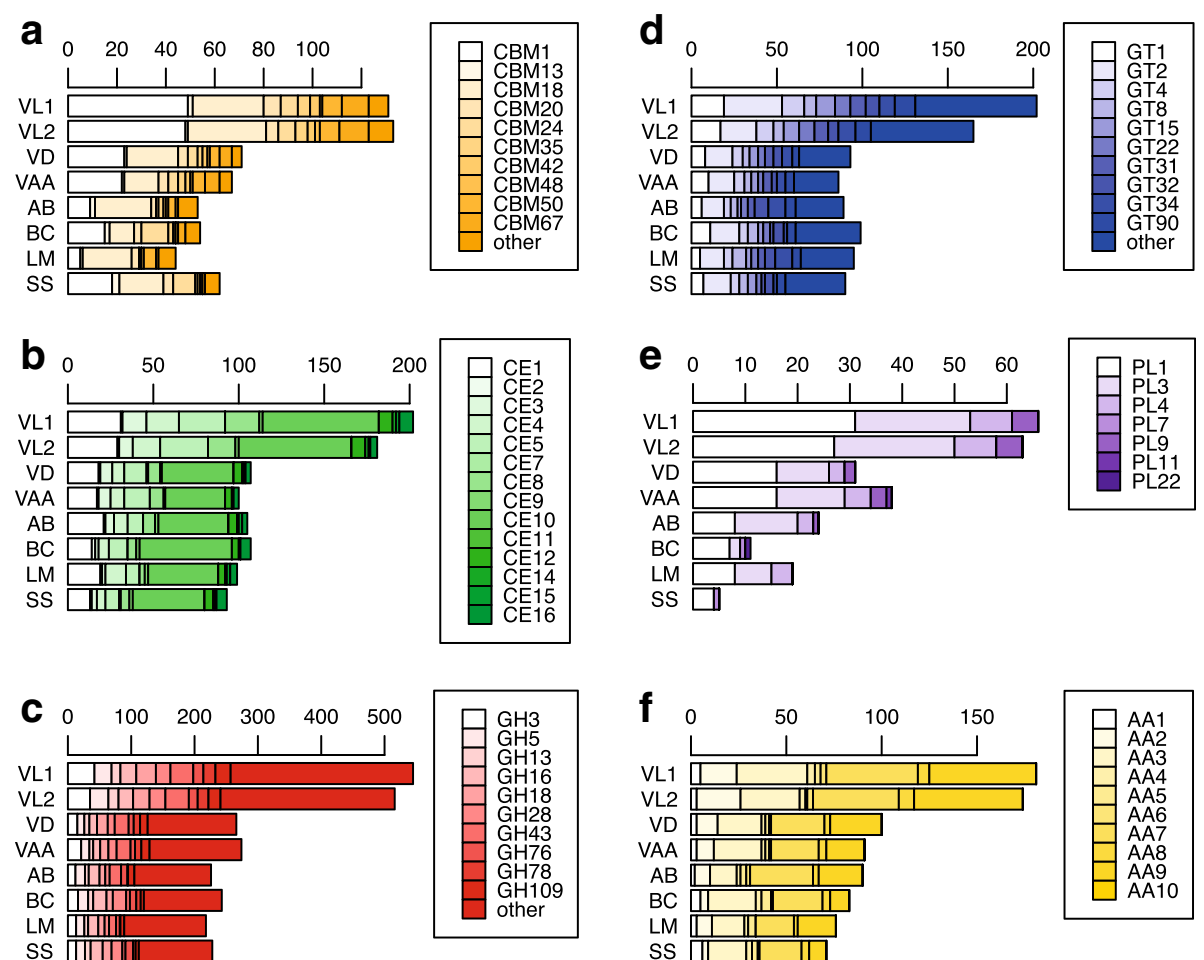

Fig. 3 Total numbers of CAZy families and domains in V. longisporum (VL1 and VL2), and selected plant pathogenic fungi. VD $=$ V. dahliae, $\mathrm{VAA}=\mathrm{V}$. albo-atrum, $\mathrm{AB}=$ Alternaria brassicicola, $\mathrm{BC}=$ Botrytis cinerea. $\mathrm{LM}=$ Leptosphaeria maculans, $\mathrm{SS}=\mathrm{Sclerotinia}$ sclerotiorum. (a) $\mathrm{CAZy}$ families with carbohydrate binding domains (CBM), (b) carbohydrate esterases (CE), (c) glycosyl hydrolases (GH), (d) glycosyl transferases $(\mathrm{GT})$, (e) pectate lyases (PL), and (f) auxiliary activities (AA). To the right, lists of individual CAZy domains

are very similar [13]. In a wider comparison of European $V$. longisporum isolates using microsatellites or simple sequence-repeat markers, Swedish and German isolates are referred to the eastern subgroup of the A1/D1 genome composition [45]. In analogy and based on new data reported here we assign the D1 lineage as having a $V$. dahliae progenitor. In our efforts to generate more information on the ancestor generating lineage A1 we estimated the distribution of synonymous substitution rate (Ks) between $V$. dahliae and the $V$. logisporum orthologous genes. The modal values of $\mathrm{Ks}$ in the $V$. dahliae to $V$. longisporum orthologous genes with $V$. dahliae origin were close to 0.025 but close to 0.1 for the genes with the hypothetical ancestor origin. For all other comparisons ( $V$. dahliae to $V$. albo-atrum, $V$. alboatrum to $V$. longisporum) the modal values of Ks were close to 0.15 . Assuming a neutral mutation rate of $1 \times 10$ ${ }^{-9}$ per site and year [46] these Ks values suggest that divergence between $V$. dahliae and $V$. albo-atrum occurred about 75 million years ago (Myr). The divergence time between $V$. dahliae and $V$. longisporum have two parts; a divergence from the part with the hypothetical ancestor lineage A1 about $50 \mathrm{Myr}$ ago and divergence from the assumed $V$. dahliae parent (D1) about 12.5 Myr ago. Hitherto we have not been able to find any sequence information that could reveal the ancestry of the lineage A1.

Hybridization events have played exceptional important roles for speciation in the host family Brassicaceae [47], which experienced ancient whole-genome duplication about 47 Myr ago [48] followed by losses of duplicated genes. Several splits and genome duplications have followed forming three lineages where today's species in this plant family can be placed. $V$. longipsorum infects species in lineage I (e.g. Arabidopsis) and lineage II (Brassica) whereas information on diseased plants from lineage III is lacking. It is premature at this stage to suggest co-evolution of ancestor Verticillium species with their host plants particularly when no sexual stage is known that could accelerate such adaptation. However, it has been suggested that the A1 lineage derive from species that used plant species in Brassicaceae as a host [19]. Notably, the first report of $V$. longisporum as a plant pathogen on Brassica crops was as recent as 1970 [49]. V. longisporum is now reported from many countries, thus the presence of different ancestor species and their fusion with potentially different $V$. dahliae isolates are not unlikely but the question on what factors that have triggered such events in this case remains unanswered. In a survey of a global collection containing 
$1120 \mathrm{~V}$. dahliae isolates, as few as 12 contained the MAT1-1 idomorph whereas 1108 had the MAT1-2 [50]. With this fragmentary knowledge, we propose that host adaptation in case of $V$. longisporum is a later event separate from the speciation process and that the sexual mode of reproduction has gone lost in the meantime. The latter switch only requires a single nucleotide change within a MAT locus [51]. Further, presence and function of transcript regulators and pheromoneassociated genes required for mating is another knowledge gap among species in the Verticillium genus.

The genomes of the vascular colonizing fungi $V$. dahliae and $V$. albo-atrum are enriched in carbohydrate modifying enzymes, particularly within the glycoside hydrolases (GH) group [20]. Their function is multifaceted, and could for example include release of cell wall carbohydrates as nutrient sources for fungal metabolism due to the hydrolysis of the glycosidic bond between carbohydrate(s) and non-carbohydrate moieties [52]. The secreted protein VdSSP1 from $V$. dahliae has been previously shown to be involved in degradation of certain plant cell-wall compounds [53], and we anticipate a similar function for several $\mathrm{GH}$ enzymes of $V$. longisporum. Many of these carbohydrate modifying enzymes are encoded in the $V$. longisporum genome but their functions are not known.

\section{Conclusions}

In this study, DNA from two single spore isolates of $V$. longisporum VL1 and VL2 were sequenced using the Illumina technology. The predicted ungapped genome size and the number of predicted protein coding genes is about double of the numbers generated from the $V$. dahliae and $V$. albo-atrum genomes [20]. A large proportion of the predicted gene models in $V$. longisporum could be partitioned into syntenic blocks with $V$. dahliae and/or $V$. albo-atrum. A hybrid genomic feature of $V$. longisporum was supported by SNP analysis. Searching for homologous genes in our VL genome data revealed two copies of MAT1-1-1 and one copy of MAT1-2-1 in both VL1 and VL2 strains encoding either the $\alpha 1$-box or the MATA_HMG domains. Extensive sequence changes have occurred in these mating genes suggesting a rather ancient descent, further supported by the overall distribution of synonymous substitution rate (Ks) between $V$. dahliae, $V$. albo-atrum and the $V$. longisporum orthologous genes. The two VL genomes analyzed in this work could be assigned to the A1/D1 nomenclature of the hybrid genome with the ancestor lineage deriving from $V$. dahliae in the D1-part, whereas the nature of A1 is unknown. Many questions remain to be answered on the $V$. longisporum genome evolution, and its host interactions. Overall, the genomic study reported here provides an important milestone of this important pathogen on Brassica crops.

\section{Methods \\ Fungal isolates and growth conditions}

The single spore isolates VL1 (CBS110220) and 43-3 from the culture collection at the Plant Biology Department, Swedish University of Agricultural Sciences (here denoted VL2), determined as $V$. longisporum by RLFP [6] and AFLP analysis [13] were used in the study. The isolates derived from diseased Brassica napus plants taken at different sites in southern Sweden, and have a modest polymorphism difference based on RFLP analysis [6]. The nuclear content of VL1 was 0.065 pg DNA and $0.051 \mathrm{pg}$ DNA in VL2, determined by flow cytometry [6]. Culture and storage of the isolates was as described earlier [6]. Fungal mycelia were used as the source for the DNA and RNA preparations.

\section{Fungal DNA and RNA preparation}

Total DNA from $V$. longisporum was extracted with Fermentas GeneJET Plant Genomic DNA Purification Mini kit (Thermo Fisher Scientific). RNA was isolated using GeneJET RNA purification kit (Thermo Fisher Scientific). Quality controls for RNA and DNA were carried out using Bioanalyzer (Agilent Techn.).

\section{Genome sequencing}

Genomic DNA was sequenced with Illumina technology and filtered from adaptor sequences and low quality reads at BGI Hong Kong (China). For each fungal sample a paired-end (200 bp insert size) and a mate-pair (5 $\mathrm{kb}$ insert size) library were used. In total 7.48 and $7.50 \mathrm{Gbp}$ high quality data were generated for VL1 and VL2, respectively. All reads were error corrected using Quake v0.3.4 [54].

\section{Mapping}

First, the Illumina reads from VL1 and VL2 were mapped to the $V$. dahliae reference genome (GCA_000400815.2), based on strain JR2 [22] using BWA-MEM v0.7.8 [55] with default settings. Variants were called jointly using Freebayes v0.9.21 (--use-best-n-alleles 5 -p 2 -hwepriors-off -haplotype-length 0) [56] and filtered using GATK VarFiltration v3.3.0 (-filter "QUAL <20.0" -filterName SAP -filter "SAP.0 > 30.0 \& \& SAP.1 > 30.0) [57]. Assuming that $V$. longisporum is a hybrid between $V$. dahliae and some other Verticillium species [3], we made an effort to differentiate between the variants originating from the different parental lineages. The mapped reads were phased using Samtools v0.1.19 (https://sourceforge.net/projects/samtools/files/samtools/0.1.19/), resulting for each $V$. longisporum lineage, a set of phased regions. Each phased region was furthermore divided whenever 
there were variants that could not be unambiguously resolved. Variants were called for the four different mapped set of $V$. longisporum reads jointly using Freebayes v0.9.21 (--use-best-n-alleles 5 -p 2 -hwe-priors-off -haplotypelength 0) as described [58] and filtered using GATK VarFiltration v3.3.0 (-filter "QUAL <20.0" -filterName SAP -filter "SAP. $0>30.0$ \& \& SAP. $1>30.0$ ). Only homozygous single nucleotide polymorphisms (SNPs) were considered from here on. For each phased region and $V$. longisporum strain, the overlapping transcripts were extracted, and modified by replacing the nucleotides in the polymorphic sites with the appropriate base for each phase. Transcripts containing internal stop codons and phased regions containing less than five transcripts were excluded from further analysis. The four-fold degenerate transversion rate $(4 \mathrm{DTv})$ between each phased $V$. longisporum transcript and the $V$. dahliae transcript was calculated using SeqinrRv3.3.1 [59]. Only regions where at least $60 \%$ of the transcripts in one phase had a larger $4 \mathrm{DTv}$ rate than in the other phase was further analyzed. This was the case for $>75 \%$ of the regions in both VL1 and VL2. Collinear blocks among $V$. dahliae, $V$. albo-atrum [20] and $V$. tricorpus [21] were identified using MCScanX [60] under default settings. The 4DTv-rate was calculated for all identified pairs of coding genes. The alignment was based on Muscle v3.8.31, and protein sequences [61] within identified collinear blocks. Whenever a comparison was made to $V$. dahliae, it was also made to VL1 and VL2 if that gene was among the set of successfully phased genes that could be partitioned into parental genomes. A maximum likelihood phylogenetic tree was created using RaXML v8.9.2 on the concatenated alignment using GTR- $\Gamma$ [62], random starting tree, rapid bootstrapping (1000 replicates) and using $V$. tricorpus as outgroup. For calculation of gene support frequency and internodal certainty gene trees were likewise generated, using the same settings but automatic bootstrap criteria (autoMRE).

\section{Assembly}

A number of assembly approaches were tried. In the end, the best de novo assemblies were generated using Velvet v1.2.9 [63] with a kmer size of 49 bp and kmer coverage cutoff at $10 \times$. The assemblies were further scaffolded using SSPACE v2.0 [64] with default settings and gap-filled using GapFiller v1.11 [65]. Mitochondrial sequences were manually identified by their coverage, GC-content, and similarity to $V$. dahliae and $V$. alboatrum mitochondria [20]. The completeness of the two genomes was assessed by mapping a set of 248 core eukaryotic genes to the de novo assembly using CEGMA [66]. Transcriptome data were generated by Illumina paired-end strand-specific sequencing of the mRNA libraries at SciLife Lab, Stockholm (Sweden). Libraries were prepared by the sequencing platform under their in-house conditions. After quality filtering using ConDeTri v2.2 [67] 113.8 and 84.08 millions high quality paired-end reads remained for VL1 and VL2, respectively. These reads were mapped to the de novo assemblies using TopHat v2.0.9 [68], assuming an intron size of 5 to $5000 \mathrm{bp}$ and otherwise default settings. The mapped reads were assembled into transcripts using Cufflinks v2.1.1 [69]. The predicted gene models could be partitioned into syntenic blocks with $V$. dahliae and/ or $V$. albo-atrum using MCScanX, and default settings.

\section{Gene annotation}

Genomes were annotated using the MAKER v2.30 pipeline [70]. First, genes were annotated based on similarity to the assembled transcripts of $V$. dahliae and $V$. alboatrum using the est2genome option. This set of genes was used to train ab-initio predictors Augustus v2.5.5 [71], Genemark-ES v2.3 [72], and SNAP [73]. In addition to ab-initio predictors and protein sequences from $V$. dahliae and $V$. albo-atrum, the assembled transcript alignments were used as evidence for the prediction. This process was iterated two times with ab-initio retraining after each prediction. Functional annotation was generated using the automated pipelines Interproscan v5.44 [74] and Blast2GO v2.5 [75] using default settings. KOG terms were assessed using the online KOG classification tool (http:// weizhonglab.ucsd.edu/ metagenomic-analysis).

\section{Repetitive sequences and transposable elements}

Repeat libraries were constructed de novo using RepeatModeler v1.0.7 (http://www.repeatmasker.org/RepeatModeler.html). The repeat library was used to mask the entire genome using RepeatMasker version open-3.0.8, with Cross_Match version 0.990329, RepBase Update 9.04 RM database version 20,040,702. For calculation of percentage of genome masked by each repeat class, the ungapped genome size was used.

\section{Secretome and candidate effectors}

Subcellular localization for all $V$. longisporum VL1 and VL2 proteins were predicted using WoLF PSORT v0.2 software [76]. The resulting putative extracellular group of proteins was further screened for the presence of signal peptides and signal peptide cleavage sites using the SignalP v4.0 program [77]. Subsequently, all proteins with signal peptides were analyzed for the presence of transmembrane domains using Phobius [78] and TMHMM version 2.0 [79]. The set of proteins with putative transmembrane domains was removed from the dataset. Remaining secreted proteins were then clustered in multiple enzymatic categories, dictated by the carbohydrate-active enzyme database, or CAZy [80]. Further divisions were made based on specific enzymatic 
groups (non-carbohydrates such as phosphatases and proteases), carbohydrate-binding capacity, and the remaining proteins were depicted as unknown. Short $(<400$ amino acids in mature chain) cysteine-rich $(>4 \%$ cysteine content in mature chain) secreted proteins were denoted as potential effectors.

Clustering of secreted proteins and potential effector proteins was assessed as follows. First the pairwise distances between all secreted proteins was calculated and binned in $5 \mathrm{~kb}$ interval. The proportion of distances in each interval was then compared to a null-distribution obtained by randomly sample $\mathrm{X}$ proteins from the total set of proteins, where $\mathrm{X}$ is the number of secreted proteins, and likewise calculate the proportion of distances in each $5 \mathrm{~kb}$ interval. The random sampling was repeated 1000 times and the 95\% confidence interval in proportion of pairwise distances in each $5 \mathrm{~kb}$ interval calculated. The process was repeated for the potential effectors.

\section{PCR and DNA sequencing}

Fifty nanograms genomic DNA from both $V$. longisporum fungal isolates (VL-1 and VL-2) was used as a template and the Phusion high fidelity DNA polymerase (Thermo Fisher) was used in the following conditions for MAT1-11 and MAT1-2-1 gene amplifications: initial denaturation at $98{ }^{\circ} \mathrm{C}$ for $30 \mathrm{~s}$ followed by 35 cycles of $98^{\circ} \mathrm{C}$ for $10 \mathrm{~s}$, $58^{\circ} \mathrm{C}$ for $30 \mathrm{~s}, 72^{\circ} \mathrm{C}$ for $1 \mathrm{~min}$, followed by final extension of $72{ }^{\circ} \mathrm{C}$ for $10 \mathrm{~min}$ before storage. Following primer sequences were used; MAT1-1-1 For: $5^{\prime}$ - ATG GAC GGT GTC CGA CCT GAAC-3' and MAT1-1-1Rev: 5' - TCA AAA GTA TGA AGC GAA CTG AGG GTGG-3' and MAT1-2-1For: $5^{\prime}$ - ATG TAT TTG TGT TCG TTA CAG ATC ACA TTTG-3' and MAT1-2-1 Rev.: 5'-CTA CAT GCT GGC CAA GAT GGC -3'. PCR products were purified from agarose gel using the GeneJET Gel Extraction Kit (Thermo Fisher), ligated to the pJET1.2/blunt vector using and cloned to E. coli DH5 $\alpha$ cells (Thermo Fisher). Positive colonies were confirmed by restriction analysis and sent for Sanger sequencing (Macrogen Inc).

\section{Phylogenetic analysis}

Maximum likelihood analysis was performed using RaXML v8.9.2, GTR $+\Gamma$ or $L G+\Gamma$ when appropriate [62], with random starting tree and rapid bootstrapping with 1000 or 10,000 replicates. Information on proteins used for the mating gene phylogeny, see Additional file 14.

\section{Additional files}

Additional file 1: Top: Distribution of coverage when mapping $V$. longisporum reads to the $V$. dahliae reference genome of strain JR2 [22]. (PDF $109 \mathrm{~kb}$ )
Additional file 2: Distribution of fourfold degenerate transversion (4DTV)-rate between homologous genes in: $V$. longisporum; $V$. longisporum and $V$. dahliae; V. longisporum and V. albo-atrum; V. dahliae and $V$. albo-atrum. (PDF $156 \mathrm{~kb}$ )

Additional file 3: Maximum likelihood phylogenetic tree using a concatenation of 3592 genes. (PDF $92 \mathrm{~kb}$ )

Additional file 4: Maximum likelihood phylogenetic trees (RAXML, model GTR+Г) for the: $\mathbf{A}$. actin (ACT) gene, B. the elongation factor 1alpha (EF) gene, $\mathbf{C}$. the glyceraldehyde-3-phosphate dehydrogenase (GP) gene, $\mathbf{D}$. the ribosomal internal transcribed spacer (ITS) region, $\mathbf{E}$. the oxaloacetate transport (OX) gene, and $\mathbf{F}$. the tryptophan synthase (TS) gene, using Verticillium spp. samples from Inderbitzin et al. [16] and homologous regions in the $V$. longisporum strain VL1 and VL2. (PDF $176 \mathrm{~kb}$ )

Additional file $\mathbf{5}$ Classes of transposable elements. (PDF 62 kb) Additional file $\mathbf{6}$ Sequence homology and Pfam domain predictions for genes illustrated in Fig. 1. (XLSX 48 kb)

Additional file 7 Predicted secreted proteins. (PDF 54 kb)

Additional file 8 Predicted secreted proteins, arranged by KOG analysis. (PDF $64 \mathrm{~kb}$ )

Additional file 9 Candidate effectors ( $<400 \mathrm{aa})$ with cysteine rich residues in VL1. (PDF $75 \mathrm{~kb}$ )

Additional file $\mathbf{1 0}$ Candidate effectors (<400 aa) with cysteine rich residues in VL2. (PDF $76 \mathrm{~kb}$ )

Additional file 11 Cysteine content of all predicted secreted proteins plotted against sequence length (amino acids) and CAZy families and domains. (PDF 409 kb)

Additional file 12 Proportion of pairwise distances between genes in $V$. longisporum VL1 and VL2 genomes binned in 5 kb intervals. (PDF 78 kb)

Additional file 13 Distribution of predicted CAZy families. (PDF 64 kb)

Additional file 14 Accession numbers for proteins used for mating gene phylogeny in Fig. 2. (PDF 112 kb)

\section{Abbreviations}

AA: Auxiliary activities; AFLP: Amplified fragment length polymorphism; CAZy: Carbohydrate active enzymes; CBM: Carbohydrate binding modules; CE: Carbohydrate esterase; CEGMA: Core eukaryotic gene mapping approach; GH: Glycoside hydrolase; GO: gene ontology; GT: glycosyl transferase; HMG: high mobility groups; KOG: eukaryotic orthologous groups; LINE: long interspersed nuclear element; LTR: long terminal repeat; MAT: mating type; NLP: necrosis and ethylene-inducing-like protein; PFAM: protein family database; RFLP: restriction length fragment polymorphism; SNP: single nucleotide polymorphism; TE: transposable elements

\section{Acknowledgements}

Not applicable

\section{Funding}

This work was supported by: the Research Council Formas, and the Swedish University of Agricultural Sciences and the following foundations: Nilsson-Ehle, Helge Ax:son Johnson, and the Memory of Oscar and Lili Lamm. Support from Science for Life Laboratory, the National Genomics Infrastructure (NGI), Sweden, the Knut and Alice Wallenberg Foundation and UPPMAX for providing assistance in DNA sequencing and computational infrastructure are acknowledged.

Availability of data and materials

Additional sequence and phylogeny data can be found in the Treebase repository (accession number: 22041).

Authors' contributions

JI, SB and AS prepared the DNA and RNA samples. JF performed all bioinformatics including database submissions. GT made the mating type analysis. JF and CD conceived the work. All authors took part in manuscript writing. All authors read and approved the final manuscript.

Ethics approval and consent to participate Not applicable. 


\section{Consent for publication}

Not applicable.

\section{Competing interests}

All authors declare that they have no competing interests.

\section{Publisher's Note}

Springer Nature remains neutral with regard to jurisdictional claims in published maps and institutional affiliations.

\section{Author details}

${ }^{1}$ Department of Plant Biology, Uppsala BioCenter, Linnean Center for Plant Biology, Swedish University of Agricultural Sciences, P.O. Box 7080, 75007 Uppsala, Sweden. ${ }^{2}$ Present Address: National Food Agency, P.O. Box 622, 75126 Uppsala, Sweden.

\section{Received: 30 June 2017 Accepted: 21 December 2017}

\section{Published online: 03 January 2018}

\section{References}

1. Pegg GF, Brady BL. Verticillium wilts. Wallingford: CABI Publ; 2002.

2. Fradin EF, Thomma BPHJ. Physiology and molecular aspects of Verticillium wilt diseases caused by V. dahliae and V. albo-atrum. Mol Plant Pathol. 2006; 7:71-86.

3. Depotter $\mathrm{J}$, Deketelaere $\mathrm{S}$, Inderbitzin $\mathrm{P}$, von Tiedemann A, Höfte $\mathrm{M}$, Subbarao KV, Wood TA, Thomma BPHJ. Verticillium longisporum, the invisible threat to oilseed rape and other brassicaceous plant hosts. Mol Plant Pathol. 2016;17:1004-16.

4. Karapapa V, Bainbridge BW, Heale JB. Morphological and molecular characterization of Verticillium longisporum comb. nov., pathogenic to oilseed rape. Mycol Res. 1997:101:1281-94.

5. Zeise K, von Tiedemann A. Morphological and physiological differentiation among vegetative compatibility groups of Verticillium dahliae in relation to V. longisporum. J Phytopathol. 2001;149:469-75.

6. Steventon LA, Fahleson J, Hu Q, Dixelius C. Identification of the causal agent of Verticillium wilt of winter oilseed rape in Sweden as Verticillium longisporum. Mycol Res. 2002;106:570-8.

7. Johansson A, Goud J-KC, Dixelius C. Plant host range of Verticillium longsporum and microsclerotia density in Swedish soils. Eur J Plant Pathol. 2006;114:139-49.

8. Johansson A, Staal J, Dixelius C. Early responses in the ArabidopsisVerticillium longisporum pathosystem are dependent on NDR1, JA- and ETassociated signals via cytosolic NPR1 and RFO1. Mol Plant-Microbe Interact. 2006;9:958-69.

9. Novakazi F, Inderbitzin P, Sandaoya G, Hauers RJ, van Tiedermann A, Subbarao KV. The three lineages of the diploid hybrid Verticillium longisporum differ in virulence and pathogenicity. Phytopathology. 2015;105: 662-73.

10. Tzelepis G, Bejai S, Sattar MN, Schwelm A, Ilbäck J, Fogelqvist J, Dixelius C. Detection of Verticillium species in Swedish soils by using real-time PCR. Arch Microbiol. 2017:199:1383-9.

11. Stark C. Das Auftreten der Verticillium-Tracheomykosen in Hamburger Garten-baukulturen. Gartenbauwissenschaft. 1961:2:493-528.

12. Jackson CW, Heale JB. Relationship between DNA content and spore volume in sixteen isolates of Verticillium lecanii and two new diploids of V. dahliae (=V. dahliae var. longisporum stark). J Gen Microbiol. 1985; 131:3229-36

13. Fahleson J, Lagercrantz U, Hu Q, Steventon LA, Dixelius C. Estimation of genetic variation among Verticillium isolates using AFLP analysis. Eur J Plant Pathol. 2003;109:361-71.

14. Fahleson J, Hu Q, Dixelius C. Phylogenetic analysis of Verticillium species based on nuclear and mitochondrial sequences. Arch Microbiol. 2004;181: 435-42

15. Zare R, Gams W, Starink-Willemse M, Summerbell RC. Gibellulopsis, a suitable genus for Verticillium nigrescens, and Musicillium, a new genus for $V$. theobromae. Nova Hedwigia. 2007:85:463-89.

16. Inderbitzin P, Davis RM, Bostock RM, Subbarao KV. Identification and differentiation of Verticillium species and $V$. Iongisporum lineages by simplex and multiplex PCR assays. PLoS One. 2013;8:e65990.
17. Inderbitzin P, Davis RM, Bostock RM, Subbarao KV. The ascomycete Verticillium longisporum is a hybrid and a plant pathogen with an expanded host range. PLoS One. 2011;6:e18260.

18. Inderbitzin P, Subbarao KV. Verticillium systematics and evolution: how confusion impedes Verticillium wilt management and how to resolve it. Phytopathology. 2014;104:564-74.

19. Depotter JRL, Seidl MF, Wood TA, Thomma BPHJ. Interspecific hybridization impacts host range and pathogenicity of filamentous microbes. Curr Opin Microbiol. 2016;32:7-13.

20. Klosterman SJ, Subbarao KV, Kang S, Veronese P, Gold SE, Thomma BPHJ, Chen Z, Henrissat B, Lee YH, Par J, Garcia-Pedrajas MD, Barbara DJ, Anchieta A, de Jonge R, Santhanam P, Maruthachalam K, Atallah Z, Amyotte SG, Paz Z, Inderbitzin P, Hayes RJ, Heiman DI, Young S, Zeng Q, Engels R, Galagan J, Cuomo CA, Dobinson KF, Ma LJ. Comparative genomics yields insights into niche adaptation of plant vascular wilt pathogens. PLoS Pathol. 2011;7: e10022137.

21. Seidl MF, Faino L, Shi-Kunne X, van den Berg GC, Bolton MD, Thomma BP. The genome of the saprophytic fungus Verticillium tricorpus reveals a complex effector repertoire resembling that of its pathogenic relatives. Mol Plant-Microbe Interact. 2015;28:362-73.

22. Faino $L$, Seidi MF, Datema E, van den Berg GCM, Janssen A, Wittenberg AHJ, $\mathrm{BPHJ}$ T. Single-molecule real-time sequencing combined with optical mapping yields completely finished fungal genome. MBio. 2015;6:e00936-15.

23. Pryszcz LP, Gabaldón T. Redundans: an assembly pipeline for highly heterozygous genomes. Nucleic Acids Res. 2016:44:e113.

24. de Jonge R, Bolton MS, van den Berg GC, Yadeta KA, Thomma BP. Extensive chromosomal reshuffling drives evolution of virulence in an asexual pathogen. Genome Res. 2013;23:1271-82.

25. Amyotte SG, Tan X, Pennerman K, Del Mar J-GM, Klosterman SJ, Ma L-J, Dobinson KF, Veronese P, et al. BMC Genomics. 2012;13:314.

26. Metzenberg RL, Glass NL. Mating type and mating strategies in Neurospora. BioEssays. 1990:2:53-9.

27. Idnurm A, Walton FJ, Floyd A, Heitman J. Identification of the sex genes in an early diverged fungus. Nature. 2008:451:193-6.

28. Martin T, Lu S-W, van Tilbeurgh H, Ripoll DR, Dixelius C, Turgeon GB, Debuchy R. Tracing the origin of the fungal a1 domain places its ancestor in the HMG-box superfamily: implication for fungal mating type evolution PLoS One. 2010:5:e15199.

29. Toruño TY, Stergiopoulos I, Coaker G. Plant-pathogen effectors: cellular probes interfering with plant defenses in spatial and temporal manners. Annu Rev Phytopathol. 2016;54:419-41.

30. Santhanam $P$, van Esse HP, Albert I, Faino L, Nürnberger T, Thomma BPHJ. Evidence for functional diversification within a fungal NEP1-like protein family. Mol Plant-Microbe Interact. 2013;26:278-86.

31. Gijzen M, Nürnberger T. Nep1-like proteins from plant pathogens: recruitment and diversification of the NPP1 domain across taxa. Phytochemistry. 2006;67:1800-7.

32. Choi J, Kim K-T, Jeon J, Lee Y-H. Fungal plant cell wall-degrading enzyme database: a platform for comparative and evolutionary genomics in fungi and oomycetes. BMC Genomics. 2013;14:S7.

33. Kubicek CP, Starr TL, Glass NL. Plant cell wall-degrading enzymes and their secretion in plant-pathogenic fungi. Annu Rev Phytopathol. 2014;52:427-51.

34. Teixeira MM, de LGP A, Kubitschek-Barreira P, Alves FL, Kioshima ÉS, AKR A Fernandes L, Derengowski LS, Ferreira KS, Souza RC, Ruiz JC, de Andrade NC, Paes HC, Nicola AM, Albuquerque P, Gerber AL, Martins VP, Peconick LD, Neto AV, Chaucanez CB, Silva PA, Cunha OL, de Oliveira FF, dos Santos TC, Barros AL, Soares MA, de Oliveira LM, Marini MM, Villalobos-Duno $H$, Cunha MM, de Hoog S, da Silveira JF, Henrissat B, Niño-Vega GA, Cisalpino PS, Mora-Montes HM, Almeida SR, Stajich JE, Lopes-Bezerra LM, Vasconcelos AT, Felipe MS. Comparative genomics of the major fungal agents of human and animal Sporotrichosis: Sporothrix schenckii and Sporothrix brasiliensis. BMC Genomics. 2014:15:943

35. Sieber CM, Lee W, Wong P, Münsterkötter M, Mewes H-W, Schmeitzl C, Varga E, Berthiller F, Adam G, Güldener U. The Fusarium graminearum genome reveals more secondary metabolite gene clusters and hints of horizontal gene transfer. PLoS One. 2014;9:e110311.

36. Irina S, Druzhinina EM, Kubicek EM, Kubicek CP. Several steps of lateral gene transfer followed by events of 'birth-and-death' evolution shaped a fungal sorbicillinoid biosynthetic gene cluster. BMC Evol Biol. 2016;16:269.

37. Revankar SG, Sutton DA. Melanized fungi in human disease. Clin Microbio Rev. 2010;23:884-928. 
38. Cannon PF, Buddie AG, Bridge PD, de Neergaard E, Lübeck M, Askar MM. Lectera, a new genus of Plectosphaerellaceae for the legume pathogen Volutella colletotrichoides. Mycokeys. 2012;3:23-36.

39. Mohanta TK, Bae H. The diversity of fungal genome. Biol Proc Online. 2015;17:8.

40. Bovers M, Hagen F, Kuramae EE, Diaz MR, Spanjaard L, Dromer F, Hoogveld $\mathrm{HL}$, Boekhout T. Unique hybrids between the fungal pathogens Cryptococcus neoformans and Cryptococcus gattii. FEMS Yeast Res. 2006;6: 599-607.

41. Morales $L$, Dujon B. Evolutionary role of interspecies hybridization and genetic exchanges in yeasts. Microbiol Mol Biol Rev. 2012;76:721-39.

42. Louis VL, Despons L, Friedrich A, Martin T, Durrens P, Casarégola S, Neuvéglise C, Fairhead C, Marck C, Cruz JA, Straub ML, Kugler V, Sacerdot C, Uzunov Z, Thierry A, Weiss S, Bleykasten C, De Montigny J, Jacques N, Jung P, Lemaire M, Mallet S, Morel G, Richard GF, Sarkar A, Savel G, Schacherer J, Seret ML, Talla E, Samson G, Jubin C, Poulain J, Vacherie B, Barbe V, Pelletier E, Sherman DJ, Westhof E, Weissenbach J, Baret PV, Wincker P, Gaillardin C, Dujon B, Souciet JL. Pichia sorbitophila, an interspecies yeast hybrid, reveals early steps of genome resolution after polyploidization. G3. 2012;2:299-311.

43. Pryszcz LP, Németh T, Gácser A, Gabaldón T. Genome comparison of Candida orthopsilosis clinical strains reveals the existence of hybrids between two distinct subspecies. Genome Biol Evol. 2014;6:1069-78.

44. Pryszcz LP, Németh T, Saus E, Ksiezopolska E, Hegedűsová E, Nosek J, Wolfe $\mathrm{KH}$, Gacser A, Gabaldón T. The genomic aftermath of hybridization in the opportunistic pathogen Candida metapsilosis. PLoS Genet. 2015;1:e1005626.

45. Depotter JRL, Seidl MF, van den Berg GCM, Thomma BPHJ, Wood TA. A previously established divergent lineage of the hybrid fungal pathogen Verticillium longisporum emerges as stem striping pathogen in British oilseed rape. bioRxiv. 2017; doi.org/10.1101/102541

46. Ossowski S, Ossowski S, Schneeberger K, Lucas-Liedó JL, Warthmann N, Clark RM, Shaw RM, Weigel D, Lynch M. The rate and molecular spectrum of spontaneous mutations in Arabidopsis thaliana. Science. 2010;327:92-4.

47. Hohmann N, Wolf EM, Lysak MA, Koch MA. A time-calibrated road map of Brassicaceae species radiation and evolutionary history. Plant Cell. 2015;27: 2770-84.

48. Kagale S, Robinson SJ, Nixon J, Xiao R, Huebert T, Condie J, Kessler D, Clarke WE, Edger PP, Links MG, Sharpe AG, Parkin IAP. Polyploid evolution of the Brassicaceae during the Cenoizoic era. Plant Cell. 2014;26:2777-91.

49. Kroeker G. Vissnesjuka på raps och rybs i Skåne orsakad av Verticillium. (disease on oilseed rape and turnip rape in Scania caused by Verticillium). Svensk Frötidning. 1970;19:10-3.

50. Short DPG, Gurung S, Hu X, Inderbitzin P, Subbarao KV. Maintenance of sexrelated genes and the co-occurrence of both mating types in Verticillium dahliae. PLoS One. 2014;9:e112145.

51. Sun S, Heitman J. Is sex necessary? BMC Biol. 2011;9:56.

52. Franková L, Fry SC. Biochemistry and physiological roles of enzymes that 'cut and paste' plant cell-wall polysaccharides. J Exp Bot. 2013;64:3519-50.

53. Liu SY, Chen JY, Wang JL, Li L, Xiao HL, Adam SM, Dai XF. Molecular characterization and functional analysis of a specific secreted protein from highly virulent defoliating Verticillium dahliae. Gene. 2013;529:307-16.

54. Kelley DR, Schatz MC, Salzberg SL. Quake: quality-aware detection and correction of sequencing errors. Genome Biol. 2010;11:R116.

55. Li H. Aligning sequence reads, clone sequences and assembly contigs with BWA-MEM. arXiv. 2013;1303:3997.

56. Garrison E, Marth G. Haplotype based variant detection from short read sequencing. arXiv. 2012;1207:3907.

57. McKenna A, Hanna M, Banks E, Sivachenko A, Cibulskis K, Kernutsky A, Garimella K, Altshuler D, Gabriel S, Daly M, DePristo MA. The genome analysis toolkit: a MapReduce framework for analyzing next-generation DNA sequencing data. Genome Res. 2010;20:1297-303.

58. Auwera GA, Carneiro MO, Hartl C, Poplin R, Del Angel G, Levy Moonshine A, Jordan T, Shakir K, Roazen D, Thibault J, Banks E, Garimella KV, Altshuler D, Gabriel S, DePristo MA. From FastQ data to high-confidence variant calls: the genome analysis toolkit best practices pipeline. Curr Protoc Bioinformatics. 2013:43:11.10.1-11.10.33.

59. Charif D, Lobry JR. SeqinR 1.0-2: a contributed package to the R project for statistical computing devoted to biological sequences retrieval and analysis. In: Bastolla U, Porto M, Roman HE, Vendruscolo M, editors. Structural approaches to sequence evolution: molecules, networks, and populations. New York: Springer Verlag; 2007. p. 207-32.

60. Wang $Y$, Tang H, Debarry JD, Tan X, Li J, Wang X, Lee TH, Jin H, Marler B, Guo H, Kissinger C, Paterson AH. MCScanX: a toolkit for detection and evolutionary analysis of gene synteny and collinearity. Nucleic Acids Res. 2012;40:e49.

61. Edgar RC. MUSCLE: a multiple sequence alignment method with reduced time and space complexity. BMC Bioinf. 2004;5:13.

62. Faircloth BC, McCormack JE, Crawford NG, Harvey MG, Brumfield RT, Glenn TC. Ultraconserved elements anchor thousands of genetic markers spanning multiple evolutionary timescales. Syst Bio. 2012;61:717-26.

63. Zerbino DR, Birney E. Velvet: algorithms for de novo short read assembly using de Bruijn graphs. Genome Res. 2008;18:821-9.

64. Boetzer M, Henkel CV, Jansen HJ, Butler D, Pirovanom W. Scaffolding preassembled contigs using SSPACE. Bioinformatics. 2011;27:78-9.

65. Boetzer M, Pirovano W. Toward almost closed genomes with GapFiller. Genome Biol. 2012;13:R56.

66. Parra G, Bradnam K, Korf I. CEGMA: a pipeline to accurately annotate core genes in eukaryotic genomes. Bioinformatics. 2007;23:1061-7.

67. Smeds L, Künstner A. ConDeTri - A content dependent read trimmer for Illumina data. PLoS One. 2011;6:e26314.

68. Kim D, Pertea G, Cole T, Pimental H, Kelley R, Salzberg SL. TopHat2: accurate alignment of transcriptomes in the presence of insertions, deletions and gene fusions. Genome Biol. 2013;14:R36

69. Trapnell C, Williams BA, Pertea G, Mortazavi A, Kwan G, van Baren MJ, Salzberg SL, Wold BJ, Pachter L. Transcript assembly and quantification by RNA-Seq reveals unannotated transcripts and isoform switching during cell differentiation. Nature Biotech. 2010:28:511-5.

70. Cantarel BL, Korf I, Robb SM, Parra G, Ross E, Moore B, Holt C, SánchezAlvarado A, Yandell M. MAKER : an easy-to-use annotation pipeline designed for emerging model organism genomes. Genome Res. 2008;18: 188-96.

71. Stanke M, Steinkamp R, Waack S, Morgenstern B. AUGUSTUS: a web server for gene finding in eukaryotes. Nucleic Acids Res. 2004;32:W309-12.

72. Lomsadze A, Ter-Hovhannisyan V, Chernoff YO, Borodovsky M. Gene identification in novel eukaryotic genomes by self-training algorithm. Nucleic Acids Res. 2005:33:6494-506.

73. Korf I. Gene finding in novel genomes. BMC Bioinf. 2004;5:59.

74. Quevillon E, Solvertoinen V, Pillai S, Harte N, Mulder N, Apweiler R, Lopez R. InterProScan: protein domains identifier. Nucleic Acids Res. 2005;33:W116-20.

75. Conesa A, Götz S, Garcia-Gómez JM, Terol J, Talón M, Robles M. Blast2GO: a universal tool for annotation, visualization and analysis in functional genomics research. Bioinformatics. 2005;21:3674-6.

76. Horton P, Park KJ, Obayashi T, Fujita N, Harada H, Adams-Collier CJ, Nakai K. WoLF PSORT: protein localization predictor. Nucleic Acids Res 2007;3:W585-7.

77. Petersen TN, Brunak S, von Heijne G, Nielsen H. SignalP 4.0: discriminating signal peptides from transmembrane regions. Nat Methods. 2011;8:785-6.

78. Käll L, Krogh A, Sonnhammer EL. Advantages of combined transmembrane topology and signal peptide prediction-the Phobius web server. Nucleic Acids Res. 2007:35:W429-32.

79. Krogh A, Larsson B, von Heijne G, Sonnhammer EL. Predicting transmembrane protein topology with a hidden Markov model: application to complete genomes. J Mol Biol. 2001;305:567-80.

80. Cantarel BL, Coutinho PM, Rancurel C, Bernard T, Lombard V, Henrissat B. The carbohydrate-active enzymes database (CAZy): an expert resource for glycogenomics. Nucleic Acids Res. 2009;3:D233-8.

\section{Submit your next manuscript to BioMed Central and we will help you at every step:}

- We accept pre-submission inquiries

- Our selector tool helps you to find the most relevant journal

- We provide round the clock customer support

- Convenient online submission

- Thorough peer review

- Inclusion in PubMed and all major indexing services

- Maximum visibility for your research

Submit your manuscript at www.biomedcentral.com/submit
) Biomed Central 